\title{
FRACTIONAL LOWER-ORDER STATISTICS FOR EFFICIENT ADAPTIVE TEMPORAL AND SPATIAL METHODS IN NON-GAUSSIAN ENVIRONMENT
}

\author{
M. Rupi and E. Del Re. \\ LENST Laboratory \\ Universitá di Firenze - Dip. Ingegneria Elettronica \\ Via di Santa Marta 3 \\ 50139 FIRENZE, Italy
}

\begin{abstract}
This paper addresses the problem of blind equalization for digitul communications using constant modulus signals in the presence of heavy-tailed additive channel noise. We compure the performance of a temporal filter antenna system with the ones obtained with an array of sensors used at the receiver to copy the information sequence. First, we demonstrate the negative effects of channel troise to the original CMA cost function in terms of reliability and convergence. Then, we introduce a now CMA criterion for both temporal and spatial systems based on the fractionul lower-order statistics (FLOS) of the received data. We perform an analytical study of the properties of the new cost function and we blustrate its convergence behavior through computer simulations.
\end{abstract}

\section{Introduction}

Adaptive chamnel equalization is an effective tool for the antenna receiver for estimating the information sequence in severe interference backgrounds. As a result, the problem of linear chamel distortion or multipath suppression has been the focus of considerable research in the signal processing and communications communities,

The Constant Modulus Algorithm (CMA) was studied by Treichler and Larimore [?] who analyzed its performance in terms of capture and lock behavior. Initial CMA studies considered only the temporal diversity at the teceiver: "The development of advanced division aceess techniques has made the concept of spatial diversity worthpursuing. As a consequence, directional array antenna beanformers have taken the place of onnidirectional antennas. In this context, Gooch and Lundel introduced the so-called constant modulus array, which exploits the constant modulus properties of the communication signal of interest to steer a beam in the ditection of the information sequense while placing nuls in the directions of interferences [1],

Most of the theoretical work on blind equalizers based on the CM criterion typically exploit higher (than second) order statistics or second-order cyclostationary statistics of the channel output signal. For this reason these methols have focused ot the case where the channel noise is assumed to follow the Gaussian modet. The Gaussian assumption is frequently motivated because il ofien leadis to mathematically tractable solutions. However, nlgorithms designed under the Gaussian assumption exhibit various degrees of perlormance degradation, depending on the non-Gaussian nature of the enviromment. For some applications, such degradation is so strong to compromise irreparably the information transmitted over such impulsive chammels, In these coses, there is a point break after which is not possible to recover the desired signal. $A$ s we will show, for the Constant Modulus Algorithm class, the presence of heavy-tail nature noise, even though not much impulsive, (i.e. realistic near Gaussian values), lead to a total lost of perfomance if used with methods optima under the Gaussianity assumption.

Indeed, experimental results have been reported where clectromngnetic noise in urban mobile-radio chamels is henyy-tailed in uature and cunnot be modeled by means of Gaussian or other exponentiat-tailed distributions $[4,5]$. In addition, impulsive chamels appeat in telephone lines, underwater acoustic communications (icc-cracks), atnospiterio efwiromments (thunderstoms), and mobile communications,

Hence, there is a need to use more general and ratistic non-Gaussian models und dosign efficient equalization techniques that take into account the possible heavy-tail nature of the data, and simultaneously work well in good Gaussian channels. Our work is devoted to the development of a novel constant modulus method which makes use of temporal filter diversity and array signal processing system for robust performance in the presence of interference/hoise envitonments that can be modeled according to the alpha-stable law. We compare the performance of a temporal FIR filter and an antenna atray model with complex cocflicients which both use a fractional lowerorder statistics on compliting the adaptation itcrative law in presence of impulsive noise and Gaussian chennels. 


\section{Symmetric AJpha-Stable Statistics for IIcavy- Tailed Noise}

Marimade as well at motural physical processes can gencrate interferences containing noise components that are impulsive in nature. If inodeling this type of signals the symmatric alpho-stable $(S \alpha S)$ distribution provides an atIractive theoretical wol. It was proven theit under broad conditions, a general elass of henvy-hiled noise follows the stable law $[6]$.

The $S \alpha S$ class of distributions is lest defined by its characteristic function:

$$
\varphi(\omega)=\operatorname{axp}\left(j \delta \omega-\gamma|\omega|^{e r}\right)
$$

where $\alpha$ is the characteristic axponent restricted to the vilues $0<n \leq 2, \delta(-\infty<\delta<\infty)$ is the location paraneter, atad $\gamma(\gamma>0)$ is the dispersion of the distribution, The dispersion parameter $\gamma$ detemines the spread of the distribution around its location parameler $\delta$, much in the same way that the variance of the Gunssian distribution cletermines the spread atound the untarl. The characteristic exponent $n$ is the most importint paraneter of the SaS distribution and it determines the shape of the distribution. The smaller the characteristic exponent $\alpha$ is, the heavier the tails of the alptha-stable clensity. It is this heavy-lail characteristic that makes the alpha-stable densities appropriatc for modeling noise that may be impulsive in nature. We should also note that the stable distribution corresponding to $\alpha=2$ cojncides with the Gerussian densily.

The appeal of $S \alpha S$ distributions as statistical models derives from some important properties. They: (i) naturally arise as limiting processes via the Generalized Central Limit Theoren: (ii) possess the stability property and share many features with the Gaussian density such as unimodality, symmetry with respeet to the location parameter, bell-shiper (iii) all but Gaussian distributions possets finte moments of orter $p$ only when $p$ is strictly less than $\alpha: \Delta|X|^{r}<\infty$ for $p<\alpha$. Stijely related with this last property, the mair concept to point out for this cless: of distribution is the introduction of fractional-lower order moments as the only possible tool capable to digitally process the analyzing data.

\section{CMA class: temporal and spatial analysis}

The constant modulus amily of blind equalizers is bused on a cost function that assigns a penalty to deviations in the modulus of the controller's complex output signal. The cost function is given by

$$
\gamma_{\rho \eta}^{O M}=E\left[|\| y(n)|\left|p^{p}-\delta\right|^{q}\right]
$$

where $E[\cdot]$ denotes statistical expectation, $y(x)$ is the controller output at time $n, p$ and $q$ are positive jutegers, and $\delta$ is a constant greater than zein related to the constant nodulus signal |2|.

The most linnous menther of this fanily is the Constant Modulus Algorithni (CMA) for which both paramelers $p$ and $a$ are equal to two. Orke pointed out the cost finctiots $J$, it is possible to approach the ipecific problemalie miter different point of vicw. Brietly spenking, a first tempral analysis was jerformeil by Treichler at al. [7], where the chennel diversity referted mainly to the lemporal domain and was produced by sampling the receivel amalog signal in tine. But spatial diversity has been used by employing at aray of sensurs at the receiver [1], showing the benefit of at spatial stmpling of the received signal.

Assuning the two diflerent signal representation, it is possible to deseribe die two distinct andlysis. Let start with the temporal approneh where a $N$ EIR fitcer whose complex coeflicients $w(n)$ are iteratively adjusted. The veetor of datil in the delay line of the filter is $\mathbf{x}(n)=$ $[n(n), \ldots, n(n-N+1)]^{\prime \prime}$, which give a complex fitte ontput ats

$$
y(n)=\mathrm{w}^{H}(n) \times(n)
$$

where the signal $x(n)$ is the received signal at the receiver al time $n$ resulting of the channe distortion and multipath elluets. Consider now an arry of $N$ equispaced sensors, which receive signals genertled by $Q$ sourees located at $v_{1}, \ldots, v_{Q}$. Assuning llo signal bandwidtl to be narow as compared to the inverse of the travel time across the arrily, it follows that, by using a complex envelop representation, he array outjut can be expressed as:

$$
\mathbf{x}(l)=\Lambda(v) \mathbf{s}(t)+\mathbf{n}(t)
$$

where $x(t)=\left[x_{1}(t), x_{2}(t), \cdots, x_{N}(t)\right]^{t^{\prime}}$ is the alraly output vector; $s(t)=\left[s_{1}(t), s_{2}(t), \cdots, s_{2}(t)\right]^{T}$ is the signal vector received by the reference sensor of the army; $\Lambda(v)$ is the $N \times Q$ steering matrix, whose rll column vector

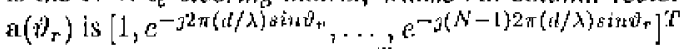
and $\mathbf{n}(t)=\left[n_{1}(t), \ldots, n_{N}(t)\right]^{N}$ is the moise wectes. The injut-output relation at the controller is the same as (3). The CMA attempts to minimize the cost lunction shown in (2) by following the path of steepest descent.

Under the bypothesis of simusoid incident signels for the temporal antlysis, assumillg $\Gamma(\omega)$ the matrix of columal vectors contuining the shifi carriers of the corresponding tone, it is possible to find it muthematical form for the complex gains of the adaplive litter for each frequencies, for both spatial and temporal dimensionalily, which is $[7,1]$

$\overline{\mathrm{v}}(n+1)=\left\{\begin{array}{cc}E^{[}\left[\Gamma^{H}(\omega) \mathrm{w}(n+1)\right] & \text { temporal analysis } \\ E\left[\Lambda^{I I}(v) w(n+1)\right] & \text { spatial inalysis }\end{array}\right.$

where the bor means the expected value, In the case of orthogonal sigunls, it is possible to find a closed form:

$$
\overline{\mathbf{v}}(n+1)=\left[\mathbf{I}-\mu\left(\begin{array}{cc}
\bar{q}_{11} & 0 \\
0 & \bar{q}_{22}
\end{array}\right)\right] \overline{\mathbf{v}}(n)
$$


where, despite of a multiplication factor of $N$ lor the tein poral analysis,

$$
\begin{aligned}
& \bar{\jmath}_{11}=\left|A_{1}\right|^{2}\left(\left|\bar{v}_{1}(n)\right|^{2}+2\left|\bar{v}_{2}(n)\right|^{2}\right) \\
& \bar{q}_{22}=\left|A_{2}\right|^{2}\left(2\left|\bar{v}_{1}(n)\right|^{2}+\left|\bar{\nu}_{2}(n)\right|^{2}\right)
\end{aligned}
$$

where we supposed a $\Gamma I R$ filter and an antemn array system with $N=2$ taps.

\section{The FI.OS-CM Time-Space Algo- rithms}

The mail elaracteristic associated will the chassical CMAs is thit they iuvolve fouth-order momenls of the signal. In the presence of heavy-triled noise, the use of sceond- or higher-onder statistics in eflect amplifies the noise. For such cases, we propose a new cost function that considers information of constent modulus regarding the communication signals and lises ]'LOS to mitigate the impulsive noise component, as we pointed out describing the SoSdistributions. The new cost function has the expression

$$
J_{p, y}^{W W O S A M A}=E\left[\left|\|y(n)\|^{(p-1)} y(n)-\delta y(n)\right|^{n}\right]
$$

where $y(n)$ is the system output and $\delta$ is a real correlated to the comstant signal modulus (actually, is the cotistant amplitude to the pth-1 power). The pair $(m, q)$ takes valucs, possibly fractional, strictly between 0 and $\alpha$, where $\alpha$ is the chamacteristic exponent of the ilpha-stitb]e distribution that best describes the statistics of the noise vector n(1). Furthemore, the product $p$ ) $a$ must be less than $a$ It is jmportant to point out the reasons why we choose to define such fractional cost function. Basically, our tools tre a transmitted signa! which has constant modulus and atl additive notse which cal be impulsive in nature: the grudient function takes into account both properties, since at the convergenee, when the amplitude should comverge to the constant value, the argument of the function tends to zero. Besides, in order to compensate the henvy tailed noise, a finctional lower-order noment ia the itcrative lanw has been inlroduced.

First, we develop the recursive update tomuila for the weighas in the temporal dimension using the principle of steepest cescent to minimize the proposed cost fuxction in (9). For the iterative law ol the complex weights vector $\mathrm{w}(n)$, sec at reference the paper [3]. Following the sane steps as for the CMA, starting with the equation (5) in order to obuin $(6)$, it is possible to write an analogons formula for the FLOS methods. Ir this case the ecjuations

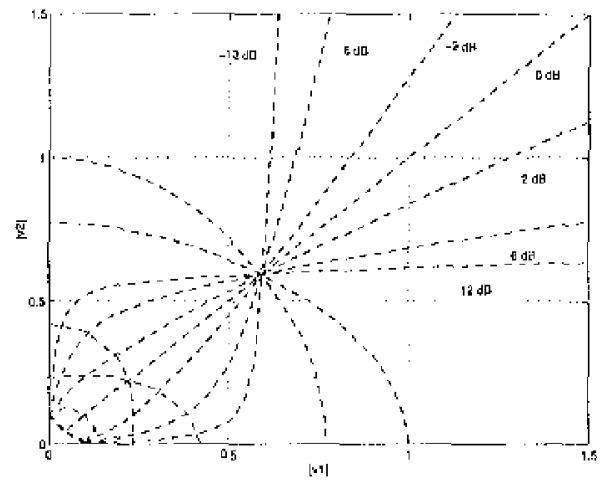

Figure 1: Broundary curves with SIR trajectories defining the lock and eaplure zones for the b'LOS-CMA.

(7) and (8) become

$$
\begin{aligned}
& \bar{q}_{11}=\left|A_{1}\right|^{2}\left(p^{P^{(n-2)}}\left\langle\left|\bar{\nu}_{1}(n)\right|^{2}+p\left|\bar{\nu}_{2}(n)\right|^{2}\right)+\right. \\
& \left.\left.-(1+n) \bar{P}^{(\mu-1-1}-1\right)\left(\left|\bar{v}_{1}(n)\right|^{2}+\left(\frac{p+1}{2}\right)\left|\bar{v}_{2}(n)\right|^{2}\right)+1\right) \\
& \bar{q}_{2 x}==\left|A_{z}\right|^{2}\left(p \bar{P}^{(p-2)}\left(p\left|\bar{v}_{\llcorner}(n)\right|^{2}+\left|\bar{\psi}_{s}(n)\right|^{2}\right)+\right. \\
& \left.-(1+p) j^{\left(\frac{\mu^{-1}}{2}-1\right)}\left(\left(\frac{p+1}{2}\right)\left|\ddot{y}_{1}(n)\right|^{2}+\left|\bar{y}_{2}(n)\right|^{2}\right)+1\right)
\end{aligned}
$$

where $P$ is the tonl ontput power: $P=\left|v_{1}(n)\right|^{2}+$ $\left|v_{2}(n)\right|^{2}$.

Naturaly, the convergenec behavior of the FLOS-CM algorithms is an impontant issue when more that one constant modulus signuls are present. It is possible to ahldress the lock and capture properties lor the array anterna system of the new criterion by finding the expression of the curve boundaries that divide the two zones in positive lack zone and positive capture zonc. Figure 1 shows the boundary curves for the FLOS-CMA and the trajectory curves for different values of Signal to Interlerence Ratio. Conparing it with the analogoss for Gaussian model in [7] it is possible to see a very close relation.

Similar studies can be carried out for the temperal analysis in cuse of simusoid signals in terms of the output weetor $\overline{\mathrm{v}}(n)$. This approach permit to opportuncly initinatize the weights vector in orler to have al priori knowledge of the at gorifitm in terms of lock (desired signal) and catpture (interference signal) behavior: One difference between the two parallel analysis is the enpability to discriminate this two lock and cuptuic zones for the cemporal system only if the incoming signals are Lones. liowever, in general the signals involved during a radio communicution transmission ale nol sinusoids, so is not possible to find an equivalent expression lor the veetor $\overline{\mathrm{v}}(n)$ as (5) for the temporal antilysis. Adversoly, in the case of antentas atray it is always possible to find an expression as descrilyed in (5) due to the presence of anothur informiltion term carried 
out with the signals represented by the angular spalial arrival direction. This is an important point which can $\mathrm{cx}^{-}$ plain the diversity in the bit error yte curves shown in the simulation result section.

\section{Experimental Results}

In this section, we test and validate the new FI.OS-CM temporal-spatial adaptive algortihms and compare their performance wilh that of the conventional $\mathrm{CM}$ algorillums in al noisy enviromment. An inlerest problem for the definition of the new cost function expressed by the equation (9), is the choice of the couple $(p, q)$. The way to choose the values $(p, q)$ is obliged to sitisfy the relation: $p \cdot q<\alpha$ duc to the characteristic of the impulsive noise. Once followed this condition, a set of possible numbers cat be chosen. A criterion which can be used to make the decision is to chose the $(p, q)$ couple which gives us a cost function $\mathrm{J}$ with better performance. Taking as exumple the case of $a=1.5$, the possible ensemble of vilues is $\{(1.1,1.1),(1.1,1.2),(1.1,1.3),(1.2,1.2),(1.2,1.1)$, $(1.3,1.1)\}$, considering the tirst decimal approximation only. Itl Figure 2 it is shown a zoom of the smallest curve for $(p, q)=(1.1,1.3)$ expanses in the bigger scale corresponding to the values $(p, q)=(1.3,1.1)$. It is so shown, that the couple $(p, q)=(1,1,1.3)$ provide better performance for the adaptive algorithm because it gives mote snooth cost function, with less curwiture and more linear trend. $N$ ll the other possible values have " bigger slope than the $(p, q)=(1.1,1.3)$ casc anyway, explaining why this choiee is the best one. Basically, the best choice is the ones which teaches a cost function as regular as possible. This property can help to lead in a stable solution, in which once we obain the desired response, we most probably will stay near, with very small oseillation movements.

I.ct consider two independent transmitted signals impinging on the array fiom directions $\theta=\left[30^{\circ},-10^{\circ}\right]$. Thic desired signal is supposed to be the first signal coming from $30^{\circ} \mathrm{deg}$ direction. The second signal is a delaycd version of the desifed one. A power control system is supposed working at the receiver. The number of snapshots available to the array is $M=100,000, M=500,000$ or $M=1,000,000$ depending on the expected BER and we performed 10 Montecarlo luns. We plot the Bit Jirtor Rate versus the SNR of the two different systems for both the original CMA and the proposed FLOS-CMA atgorilluns: the ones with temporal analysis and the second with spatial processing (cf. Figure 3). in Figure 3 (a) the noise component is modeled as an alpha-stable process witl $\alpha=1.85$, 1.e., the notse is fairly close to Gaussiall. Actualy, this value has been measured for some paticular communication transmissions, Figure 3 (b) is the bit crror tate of a Gaussian chamel.

Figure 3 demonstrates that ocedrences of noise oullicrs

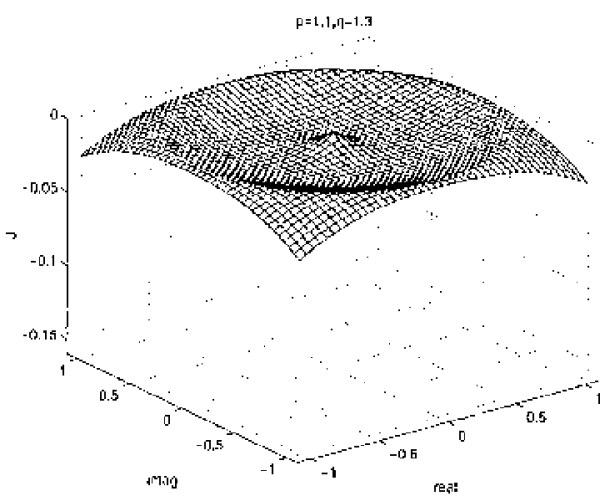

(a)

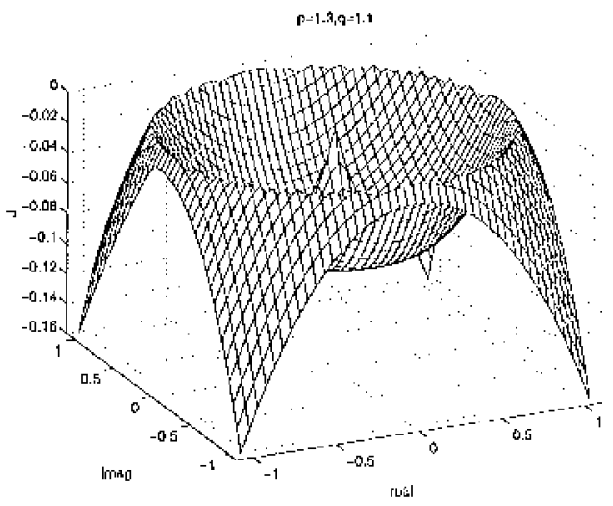

(b)

Figne 2: FI, OS-CMA Cost Function, $(p, 9)=(1.1,1,3)(a)$ $(p, q)=(1,3,1,1)(b)$.

during the adaptition, have an adverse affect to the learning curve of the original $\mathrm{CMA}$ method. In fact, even a relative low impulsiveness of $\alpha=1.8$ s has a disruptive elfect on the convergenee helavior of the classical CM algorithms, both in the temporal and spatial base, Bricfly speaking, as an impulse occurs, the elassical CM algolibums lonke their convergence properties and they become not able anymote to recover the trumsinted information serfuence On the other band, the proposed [i. OS-CMA cost function can strppress the noise components and results in it much deeper bit ertor rate curve. The gain that wo cin teacla between the array model and the FIR system wan be even of 10 ald for low $S N R(4,6,8)$ and becomes smoother as the SNR increases (few dB).

However, in presence of Gaussian noise channels, the CM algoritlims outperlorn the Fl.OS methods ranging from values between 2 and $8 d B$. $\Lambda$ s we can see from the curves, the antenna array system at the receiver sliows al consistent improvement in terms of ntmber of bit correely receivel dne to a further diversity that suct nodel 


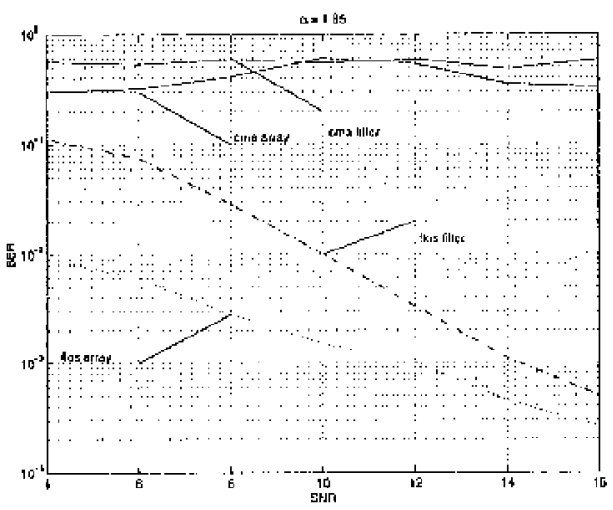

(a)

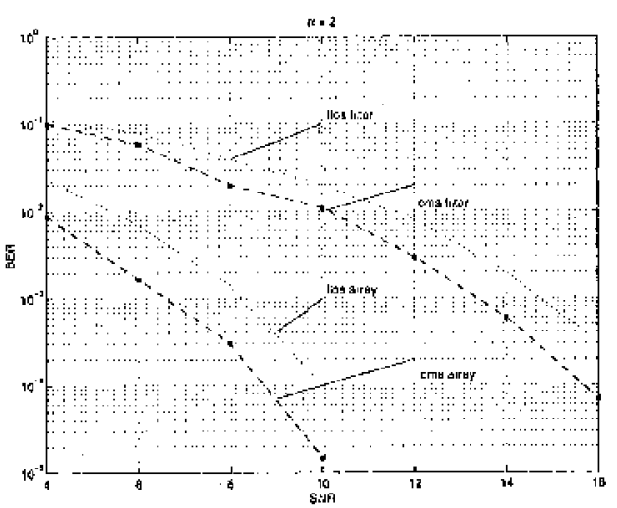

(b)

Figure 3: Bit error rate curves for the filter and the array receiver system, (a): $\alpha=1.85$, (b): $\alpha=2$.

implement. Besicles, as pointed ont in the previous section, the impossibility to clefine the two lock and capture zones in the case of the $N$ dimensionality FIR filter for all radio communications signals strongly bounds the performance results of the proposed FLOS-CM algoritlon in the case of temporal amalysis.

\section{Conclusions}

We proposed a now method for blind equalization of conmunication signals using a constant moduins criterion based on froctional lower-urder statistics. The introduced FLOS-CM Algorithms exploit the constant modulus property of the signal of interest and uses the heavytailed noise suppression capabilities of FLOS to recover the information sequence in at temporal and spatial point of view. The cupability of an array system to stecr a beam in the direction of the signal while suppressing interference and noise is resulting in a better performatice in terms of bit error rate. Besides, the directional of arrival informalion allows the algorithm to divide the signal space in the capture and lock zone. Such property, which is nol always possible with a FIR omnidirectional filter, provides a further improvement in the l3ER cutves.

The main advantage of the proposed method is its robustness in the presence of varions noise environments. Truly, by changing lae parameters $\gamma$ and $q$ in the criterion iil (9) we obtain a class of lLOS-based $C M$ Algorithms which provide considerable tlexibility that can be useful for oplimization purposes in the presence of non stationary noise environments. Besides, we showed a similar behavior of CMA and FLOS algarillums in presence of Gaussian chtinucls.

The proposed method developed using t'LOS has approximately the same computalionil complexity as the existing CMA nethods. The additional conputationat lout is cue to the need tor calculating al frational power $(p<2$ ) tather than a square power. The technique can bo used in commereial communication applications in which impulstwe chaniles tend to produce largo-amplitude interferences and sharp noise spikes more frequently than what is expected from Gaussian channels.

\section{References}

[i] R, Gooch and J. Lundell. The cm arrily: an adaptive beamformer for constant modulus signals. In Procaedings of Intemational Conference on Acoustic, speech, and Signal Processing, 1986.

(2) M.G. Larimore and J.R. Treichler. Convergence behavior of the conslant modulus algorithm, In International Conference on Acoustic, Speech, and Signal Processing, 1983.

[3] M. Rupi, P. 'J'sakalides, C. L. Nikias, and R. Del Re. Robust constant modulus atrays based on liactional lower-order statistics. WEE /CASSP, pag. 2945-2948, Phoenix, 15-19 Mnr. 1999.

[4] D. Middlelon, Statistical-phisical models of urban radio noise etrvironment - patt $\mathrm{i}$ : Foundations, fEEE Trans. Clect. Comput 14:38-56, 1972.

[5] U. Mitra and H.V. Poor. Detection of spreid-spectrum signals in multi-user emironment. In Proc. of IEEE ICASSP, pages 1844-18\$7, Detroit, Michigan, May 9.12, 1995 .

[6] C L Nikias and M Shao, Signal Processing with Alpha-Stable Distributions and Applications. John Wiley and Sons, New York, 1995.

[7] J.R. Treichler and M.G. Larimore. The tone cipture propetties of cma-based interfercnee suppressors. IEEE Transactions on Acoustic, Speech, and Signal Processing, Allg. 1985. 